Draft VERSION August 4, 2016

Preprint typeset using LATE $_{\mathrm{E}} \mathrm{X}$ style AASTeX6 v. 1.0

\title{
A MAGNETAR-LIKE OUTBURST FROM A HIGH-B RADIO PULSAR
}

\author{
R. F. Archibald, V. M. Kaspi, S. P. Tendulkar, and P. Scholz \\ Department of Physics and McGill Space Institute, McGill University, 3600 University St., Montreal QC, H3A 2T8, Canada \\ Email: rarchiba@physics.mcgill.ca
}

\begin{abstract}
Radio pulsars are believed to have their emission powered by the loss of rotational kinetic energy. By contrast, magnetars show intense X-ray and $\gamma$-ray radiation whose luminosity greatly exceeds that due to spin-down and is believed to be powered by intense internal magnetic fields. A basic prediction of this picture is that radio pulsars of high magnetic field should show magnetar-like emission. Here we report on a magnetar-like X-ray outburst from the radio pulsar PSR J1119-6127, heralded by two short bright X-ray bursts on 2016 July 27 and 28 (Kennea et al. 2016; Younes et al. 2016). Using Target-of-Opportunity data from the Swift X-ray Telescope and NUSTAR, we show that this pulsar's flux has brightened by a factor of $>160$ in the $0.5-10 \mathrm{keV}$ band, and its previously soft X-ray spectrum has undergone a strong hardening, with strong pulsations appearing for the first time above $2.5 \mathrm{keV}$, with phase-averaged emission detectable up to $25 \mathrm{keV}$. By comparing Swift-XRT and NuSTAR timing data with a pre-outburst ephemeris derived from Fermi Large Area Telescope data, we find that the source has contemporaneously undergone a large spin-up glitch of amplitude $\Delta v / v=5.74(8) \times 10^{-6}$. The collection of phenomena observed thus far in this outburst strongly mirrors those in most magnetar outbursts and provides an unambiguous connection between the radio pulsar and magnetar populations.
\end{abstract}

\section{INTRODUCTION}

PSR J1119-6127 is a radio pulsar having spin period $P=0.407 \mathrm{~s}$, discovered in the Parkes multibeam $1.4-\mathrm{GHz}$ survey (Camilo et al. 2000). The pulsar's $P$ and spin-down rate $\dot{P}=4.0 \times 10^{-12}$ imply a characteristic age $\tau<2 \mathrm{kyr}$, making this object one of the youngest pulsars in the Galaxy, consistent with an association with the supernova remnant G292.2-0.5 (Crawford et al. 2001) at a distance of $8.4 \mathrm{kpc}$ (Caswell et al. 2004). Those same spin parameters, under the assumption of vacuum dipole spin-down, imply a dipolar surface magnetic field $B=4.1 \times 10^{13} \mathrm{G}$, among the highest known among radio pulsars. The pulsar's spin-down luminosity is $\dot{E}=2.3 \times 10^{36} \mathrm{erg} \mathrm{s}^{-1}$.

Past X-ray observations of the source (Gonzalez \& SafiHarb 2003; Safi-Harb \& Kumar 2008; Ng et al. 2012) have shown it to be a soft X-ray pulsar, with strong pulsations below $2.5 \mathrm{keV}$, and none seen above this energy. This emission was well described by a two-component model consisting of a power law of index $\sim 2.1$, with a hot thermal component of blackbody temperature $k T \simeq 0.2 \mathrm{keV}$, high compared to lower-field radio pulsars of comparable age (see also Kaspi \& McLaughlin 2005; Zhu et al. 2011; Olausen et al. 2013). Radio pulse profile changes, short radio bursts and unusual timing recoveries were observed near epochs of glitches in this source (Weltevrede et al. 2011; Antonopoulou et al. 2015), reminiscent of radio radiative behavior (e.g. Camilo et al. 2006) and glitch recoveries (Dib \& Kaspi 2014) following magnetar outbursts. The pulsar is also among the highest-
B sources to have been detected in $\gamma$-rays by Fermi (Parent et al. 2011).

The Fermi Gamma-ray Burst Monitor (GBM) and Swift Burst Alert Telescope (BAT) both reported short magnetarlike bursts from PSR J1119-6127, on 2016 July 27 (UT 13:02:08; Younes et al. 2016) and 2016 July 28 (UT 01:27:51; Kennea et al. 2016), respectively. Immediately following the BAT burst, the Swift X-ray Telescope (XRT) found a bright X-ray source at the position of PSR J1119-6127 (Kennea et al. 2016) with pulsations at the rotational period (Antonopoulou et al. 2016). This suggests that this radio pulsar has had a magnetar-like outburst, similar to the 2006 transition of rotation-powered, but radio-quiet, pulsar PSR J1846-0258 (Gavriil et al. 2008). Interestingly, the radio pulsations from PSR J1119-6127 have disappeared (Burgay et al. 2016).

We report here on our Target-of-Opportunity (ToO) X-ray observations of PSR J1119-6127 with the Swift/XRT and NUSTAR during the first few days of the transition, as well as on pre-outburst Fermi/LAT timing data.

\section{OBSERVATIONS \& ANALYSIS}

\subsection{Swift-XRT \& NuSTAR Observations}

The Swift-XRT (Burrows et al. 2005) slewed to observe PSR J1119-6127 $62.8 \mathrm{~s}$ after the BAT trigger (Kennea et al. 2016). XRT was operated in Photon Counting (PC) mode for this 2.2-ks observation (ObsID 00706396001, spanning 2016 July 28 01:28 - 02:07 UT), and in Windowed- 
Timing (WT) mode for the follow-up observations (ObsID 00033448001/2), spanning July $2817: 20$ to July 29 03:11 UT and July 31 04:20 to August 1 20:37 UT for exposures of 9.9 and $4.8 \mathrm{ks}$, respectively.

As the time resolution of PC mode is $2.5 \mathrm{~s}$ (longer than the period of the pulsar), only WT mode observations, with a time resolution of $1.76 \mathrm{~ms}$, were used in the timing analysis.

NuSTAR (Harrison et al. 2013) began ToO observations of PSR J1119-6127 at 2016 July 28, 23:05:12 UT yielding a total exposure time of $54.5 \mathrm{ks}$ (ObsID 80102048002) partially overlapping with XRT observation 00033448001. The data from the two focal plane modules of NUSTAR are referred to hereafter as FPMA and FPMB.

The Swift-XRT and NuSTAR data were processed with the standard xrtpipeline and nupipeline scripts, respectively, using HEASOFT v6.17 and time corrected to the Solar System barycenter from the Chandra location of PSR J11196127 (Gonzalez \& Safi-Harb 2003).

For Swift, we selected only Grade 0 events for spectral fitting as other event grades are more likely to be caused by background events (Burrows et al. 2005). Swift spectra were extracted from the selected regions using extractor. Source photons were extracted from a 10-pixel radius circular region centered on PSR J1119-6127 with an annular background region with an inner and outer radius of 75 and 125 pixels, respectively.

The WT observations had multiple soft X-ray bursts which appear in both the source and background regions that, from past experience, seem to be instrumental in origin. Hence, for the WT mode data, we excluded all photons below $0.7 \mathrm{keV}$ from our analysis.

For NUSTAR the source events were extracted within a 30pixel $\left(72^{\prime \prime}\right)$ radius around the centroid. Appropriate background regions were selected to be on the same detector as the source location. Spectra were extracted using the nuproducts script.

Using grppha, channels 0-70 (<0.7 keV) and 700-1023 $(>7 \mathrm{keV})$ for Swift data and channels $0-35(<3 \mathrm{keV})$ and 1935-4095 (> 79 keV) for NuSTAR data were ignored and all good channels were binned to have a minimum of one count per energy bin.

\subsection{Fermi Large Area Telescope Observations}

We downloaded Pass 8R2 events of Fermi Large Area Telescope (LAT) (Atwood et al. 2009) all-sky survey observations from 2008 August 4 to 2016 July 30 from a one degree radius surrounding the Chandra position of PSR J11196127 and applied the recommended event selection. In the timing analysis, we used only photons having energy greater that $500 \mathrm{MeV}$ based on the $\gamma$-ray pulse profile of PSR J11196127 (Parent et al. 2011). We corrected the arrival times of each photon to the Solar System barycenter using the tempo2 fermi plug-in (Ray et al. 2011).

\section{RESULTS}

\subsection{Timing Analysis}

Times-of-arrivals (TOAs) of $\gamma$-ray pulses were extracted using a maximum likelihood (ML) method, described in Livingstone et al. (2009). We extracted a TOA from photons collected in every 100 day span as a trade-off between TOA spacing and precision. We extended the ephemeris presented by Antonopoulou et al. (2015) using the LAT detected photons until the GBM-detected burst (Younes et al. 2016). We present a phase-coherent ephemeris in Table 1 and the timing residuals in the left panel of Figure 1.

To determine an ephemeris for the post-outburst Swift and NuSTAR observations, we folded the soft X-ray photons (< $10 \mathrm{keV}$ ) from each observation, starting with the ephemeris from the LAT observations and extracted TOAs from each orbit using the ML method. As there is no apparent evolution in the pulse profiles over the $<10 \mathrm{keV}$ energy band, the offset between TOAs from both telescopes should be minimal. We then used the tempo2 timing software package (Hobbs et al. 2006) to fit the TOAs.

It is apparent that the LAT ephemeris did not accurately describe the post-outburst TOAs and requires a change in the spin frequency. Due to the long integration times required to extract a TOA from LAT, we are unable to constrain the exact glitch epoch; for this analysis, we have fixed the glitch epoch to the time of the first GBM-detected burst (Younes et al. 2016) and fitted for a glitch in spin frequency and frequency derivative. We measure a spin-up glitch with $\Delta v=1.40(2) \times 10^{-5} \mathrm{~Hz}$ and $\Delta \dot{v}=-1.9(5) \times 10^{-12} \mathrm{~Hz} \mathrm{~s}^{-1}$. We caution that this represents a snapshot of the frequency evolution, and that following glitches in magnetars, complex recoveries are often observed (e.g. Dib et al. 2008; Dib \& Kaspi 2014).

In Figure 2 we show the soft $(0.7-2.5 \mathrm{keV})$, and hard (2.5$10 \mathrm{keV}) \mathrm{X}$-ray pulse profiles from the radio pulsar state in 2011 ( $\mathrm{Ng}$ et al. 2012), and the magnetar-like state in 2016. In the soft band, the root-mean-squared (RMS) pulse fraction has increased from $38(3) \%$ to $71(4) \%$, while the pulse shape has remained similar. In the hard band, the RMS pulse fraction went from $<10 \%$ to $56(3) \%$ pulsed.

\subsection{X-ray Spectroscopy}

All X-ray spectra were fit using XSPEC v12.9.0 (Arnaud 1996) with a common value for hydrogen column density $\left(N_{\mathrm{H}}\right)$. Magnetar spectra are typically described with an absorbed blackbody plus power-law model, which we use here. However, the independent Swift-XRT observations 00706396001 and 00034632002 were fit with a simple absorbed blackbody model between $0.7-7 \mathrm{keV}$ as there is no constraint on the power law without the NuSTAR spectrum and there is little power-law contribution below $7 \mathrm{keV}$.

We used Cash statistics (Cash 1979) for fitting and parameter estimation of the unbinned data. $N_{\mathrm{H}}$ was fit using wilm 


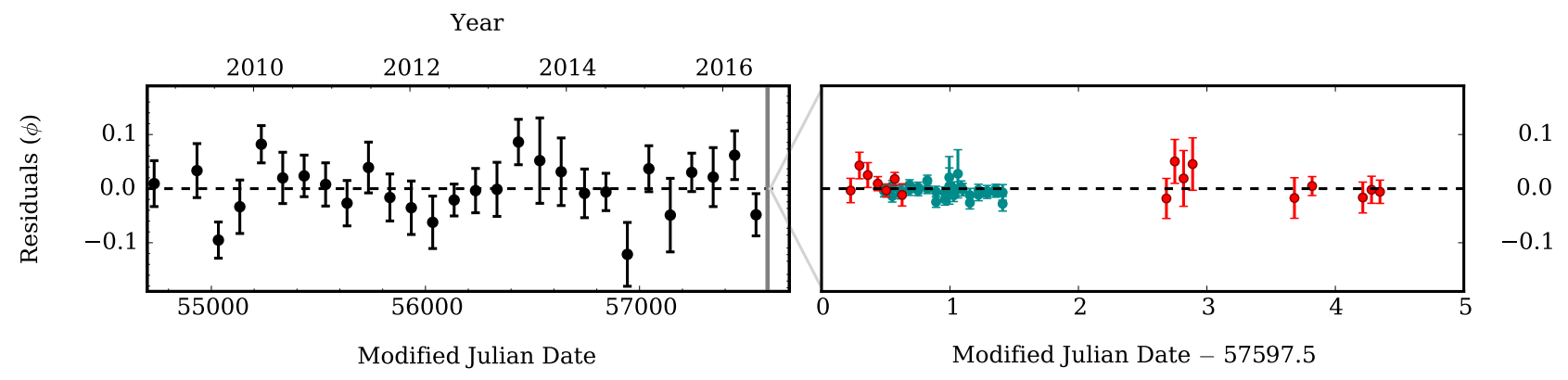

Figure 1. The left panel shows the LAT timing residuals of PSR J1119-6127 from MJD 54732 to 57544, (2008 September 23 - 2016 June 5), for the solution presented in Table 1. The gray vertical bar shows the burst epoch. The right panel shows the residuals for the post-outburst timing solution. Black points are LAT, red are XRT, and cyan are NuSTAR.

Table 1. Phase-Coherent Ephemeris for PSR J1119-6127.

\begin{tabular}{|c|c|}
\hline \multicolumn{2}{|c|}{ Fermi LAT Ephemeris } \\
\hline Dates (MJD) & $54732.82-57544.08$ \\
\hline Dates & 2008 Sept $23-2016$ June 5 \\
\hline Epoch (MJD) & 56264.00000 \\
\hline$v\left(\mathrm{~s}^{-1}\right)$ & $2.4425792940(9)$ \\
\hline$\dot{v}\left(\mathrm{~s}^{-2}\right)$ & $-2.390210(4) \times 10^{-11}$ \\
\hline$\ddot{v}\left(\mathrm{~s}^{-3}\right)$ & $5.68(3) \times 10^{-22}$ \\
\hline$\dddot{v}\left(s^{-4}\right)$ & $-1.46(9) \times 10^{-30}$ \\
\hline$v^{(4)}\left(\mathrm{s}^{-5}\right)$ & $-3.1(7) \times 10^{-38}$ \\
\hline$v^{(5)}\left(\mathrm{s}^{-6}\right)$ & $1.8(1) \times 10^{-45}$ \\
\hline$v^{(6)}\left(\mathrm{s}^{-7}\right)$ & $3(1) \times 10^{-53}$ \\
\hline RMS residual (ms) & 15.5 \\
\hline RMS residual (phase) & 0.037 \\
\hline$\chi_{v}^{2} / \mathrm{dof}$ & $1.08 / 20$ \\
\hline \multicolumn{2}{|c|}{ Post-Outburst Ephemeris } \\
\hline Dates (MJD) & $57597.72-57601.85$ \\
\hline Dates & 28 July -1 Aug 2016 \\
\hline Epoch (MJD) & 57596.547 \\
\hline$v\left(\mathrm{~s}^{-1}\right)$ & $2.43983734(8)$ \\
\hline$\dot{v}\left(\mathrm{~s}^{-2}\right)$ & $-2.57(5) \times 10^{-11}$ \\
\hline RMS residual (ms) & 4.22 \\
\hline RMS residual (phase) & 0.001 \\
\hline$\chi_{v}^{2} / \mathrm{dof}$ & $0.74 / 46$ \\
\hline \multicolumn{2}{|c|}{ Glitch Parameters } \\
\hline Glitch Epoch (MJD), fixed & 57596.547 \\
\hline$\Delta v\left(\mathrm{~s}^{-1}\right)$ & $1.40(2) \times 10^{-5}$ \\
\hline$\Delta \dot{v}\left(\mathrm{~s}^{-2}\right)$ & $-1.9(5) \times 10^{-12}$ \\
\hline
\end{tabular}

Note: Figures in parentheses are the $1 \sigma$ TEMPO2 uncertainties in the least-significant digits quoted. The source location was fixed at the Chandra position.

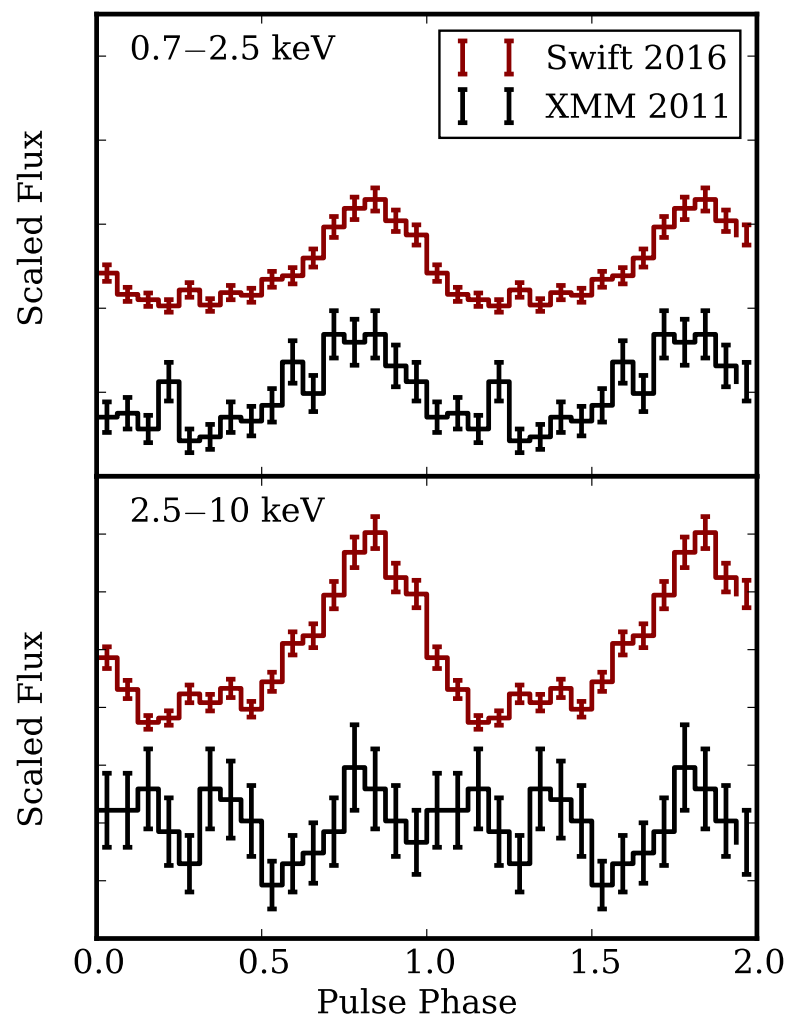

Figure 2. Pre- and post-outburst X-ray pulse profiles of PSR J11196127 from Swift-XRT (00034632001, red) and 2011 XMM-Newton data ( $\mathrm{Ng}$ et al. 2012) (black) in the soft (0.7-2.5 keV, top panel) and hard (2.5-10 keV, bottom panel). The profiles have been arbitrarily offset vertically for clarity and aligned in phase based on the soft $\mathrm{X}$-ray profile.

abundances and vern photoelectric cross-sections. The normalizations of NuSTAR FPMB and Swift-XRT spectra were allowed to vary with respect to that of the NuSTAR FPMA spectrum.

The hard X-ray tail seen above $8 \mathrm{keV}$ in the PC mode observation (00706396001) may be caused by contamination 


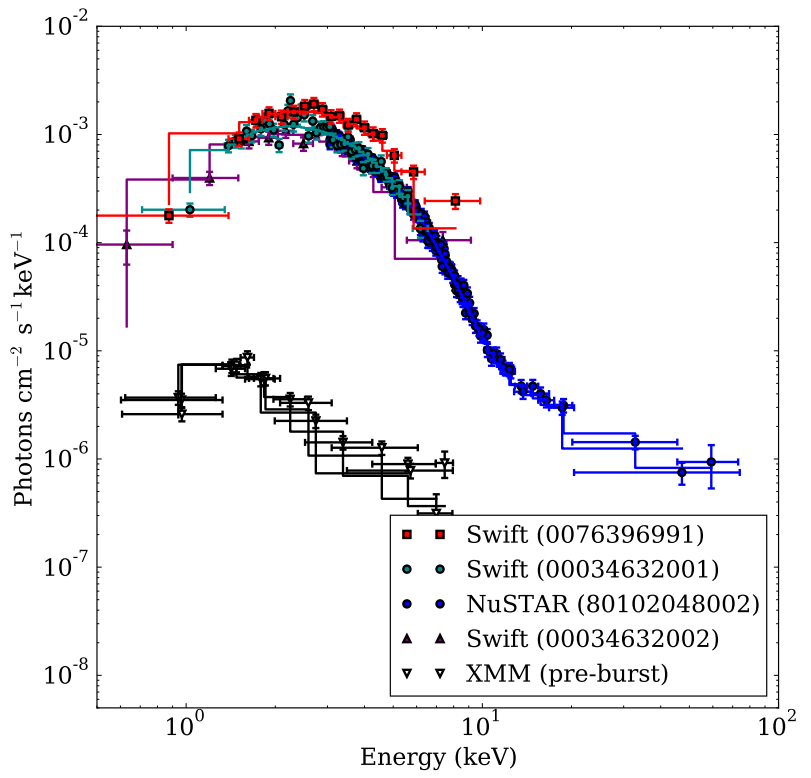

Figure 3. X-ray spectra of PSR J1119-6127. The data are as follows: pre-outburst XMM-Newton spectrum (inverted black triangles), Swift-XRT spectrum at burst (red squares), Swift-XRT and NUSTAR spectrum one day after the burst (green and blue circles, respectively), and a Swift-XRT spectrum three days after the burst (purple triangles). The flux increase above $8 \mathrm{keV}$ in the Swift-XRT burst spectrum is likely due to pileup (see Section 3.2 for details). For clarity, the NUSTAR spectra from FPMA and FPMB are combined.

due to short temporally unresolved X-ray bursts. Magnetarlike bursts are intrinsically harder than the average spectrum and the high count-rate leads to pile-up effects within the 2.5s CCD readout time (e.g. Scholz \& Kaspi 2011). These pileup effects are not mitigated by standard techniques such as the removal of the central bright region, as they are bunched temporally rather than spatially. If the hard X-ray tail were indeed real, it would have needed to fade by $\sim 2$ orders of magnitude in the day before the NUSTAR pointing to be consistent with the measured hard X-ray flux, while the blackbody temperatures measured on the two epochs are consistent with slow cooling. Hence, to fit the average spectra, we truncate the 00706396001 spectra above $7 \mathrm{keV}$.

Figure 3 shows the current Swift $+N u S T A R$ spectral fits in comparison with a pre-burst XMM-Newton spectrum (from $\mathrm{Ng}$ et al. 2012). Table 2 details the parameter values with $90 \%$ confidence error bars.

Immediately after the burst, we measure a blackbody temperature $k T=1.10(6) \mathrm{keV}$, slightly decreasing to $0.96(1) \mathrm{keV}$ and $0.93(1) \mathrm{keV}$ in the follow-up spectra. This is substantially higher than the pre-burst blackbody temperature of $0.2 \mathrm{keV}$ ( $\mathrm{Ng}$ et al. 2012). In the NUSTAR spectra, we also measure a hard power law with photon index $\Gamma=1.2(2)$ that is significantly harder than the pre-burst value $\Gamma_{\mathrm{PSR}}=$ 2.1(8) (Ng et al. 2012). We also note that $\mathrm{Ng}$ et al. (2012) and
Safi-Harb \& Kumar (2008) measured the power law emission from the pulsar wind nebula (PWN) around PSR J1119-6127 to have $\Gamma_{\mathrm{PWN}}=1.1-1.4$, close to the current hard power law index, but with a flux of $\sim 2 \times 10^{-14} \mathrm{erg} \mathrm{cm}^{-2} \mathrm{~s}^{-1}$, almost three orders of magnitude fainter.

Table 2. Spectral Fits to NuSTAR and Swift-XRT Data

\begin{tabular}{|c|c|c|}
\hline Component & Parameter & Value \\
\hline tbabs & $N_{\mathrm{H}}\left(10^{22} \mathrm{~cm}^{-2}\right)$ & $1.2 \pm 0.1$ \\
\hline \multicolumn{3}{|c|}{ Swift-XRT 0076396991 (tbabs *bbody) } \\
\hline bbody & $k T_{\mathrm{BB}}(\mathrm{keV})$ & $1.10 \pm 0.06$ \\
\hline C-Stat/dof & & $333.82 / 413$ \\
\hline goodness $^{\mathrm{a}}$ & & $12 \%$ \\
\hline Flux $(0.5-10 \mathrm{keV})^{\mathrm{b}}$ & & $4.1 \pm 0.1$ \\
\hline$L_{X}(0.5-10 \mathrm{keV})^{\mathrm{c}}$ & & 3.5 \\
\hline \multicolumn{3}{|c|}{$\begin{array}{l}\text { NuSTAR } 80102048002+\text { Swift-XRT } 00034632001 \\
\text { const * tbabs* (bbody+powerlaw) }\end{array}$} \\
\hline \multirow[t]{2}{*}{ const } & $C_{\text {FPMB }}{ }^{\mathrm{d}}$ & $1.01 \pm 0.02$ \\
\hline & $C_{\mathrm{XRT}}{ }^{\mathrm{d}}$ & $0.94 \pm 0.04$ \\
\hline bbody & $k T_{\mathrm{BB}}(\mathrm{keV})$ & $0.96 \pm 0.01$ \\
\hline powerlaw & $\Gamma$ & $1.2 \pm 0.2$ \\
\hline C-Stat/dof & & $2133.1 / 2327$ \\
\hline goodness $^{\mathrm{a}}$ & & $20 \%$ \\
\hline Flux $(0.5-10 \mathrm{keV})^{\mathrm{b}}$ & & $2.7 \pm 0.1$ \\
\hline$L_{X}(0.5-10 \mathrm{keV})^{\mathrm{c}}$ & & 2.3 \\
\hline Flux $(3-79 \mathrm{keV})^{\mathrm{b}}$ & & $1.9 \pm 0.1$ \\
\hline$L_{X}(3-79 \mathrm{keV})^{\mathrm{c}}$ & & 1.6 \\
\hline \multicolumn{3}{|c|}{ Swift-XRT 00034632002 (tbabs *bbody) } \\
\hline bbody & $k T_{\mathrm{BB}}(\mathrm{keV})$ & $0.93 \pm 0.06$ \\
\hline C-Stat/dof & & $304.7 / 355$ \\
\hline goodness $^{\mathrm{a}}$ & & $34 \%$ \\
\hline Flux $(0.5-10 \mathrm{keV})^{\mathrm{b}}$ & & $2.1 \pm 0.2$ \\
\hline$L_{X}(0.5-10 \mathrm{keV})^{\mathrm{c}}$ & & 1.8 \\
\hline
\end{tabular}

\footnotetext{
${ }^{a}$ Percentage of C-Stat statistic simulation trials from model parameters that are less than the fit statistic.

${ }^{b}$ Unabsorbed flux in units of $10^{-11} \mathrm{ergcm}^{-2} \mathrm{~s}^{-1}$.

${ }^{c} \mathrm{X}$-ray luminosity assuming isotropic emission at a distance of $8.4 \mathrm{kpc}$ in units of $10^{35} \mathrm{erg} \mathrm{s}^{-1}$.

$d_{\text {Cross-normalization constants w.r.t. NUSTAR FPMA. }}$
}

\section{DISCUSSION}

The outburst from PSR J1119-6127 is observationally very similar to those seen in magnetars. The phenomenology of magnetar outbursts is rich (see Rea \& Esposito 2011, for a review), but with established commonalities, practically all of which are observed in the PSR J1119-6127 event. A hallmark of magnetar outbursts are short-duration $(<1$-s) hard 
X-ray bursts, as reported for PSR J1119-6127 (Kennea et al. 2016; Younes et al. 2016). The large flux enhancement, here by a factor of $>160$, is commonly seen in magnetar outbursts, notably those in which the quiescent luminosity is below $\sim 10^{33} \mathrm{erg} \mathrm{s}^{-1}$ (e.g. Scholz \& Kaspi 2011; Kargaltsev et al. 2012). Moreover, the spectral hardening we report is classic for magnetar outbursts (Rea \& Esposito 2011), as are timing anomalies, most often spin-up glitches (Dib et al. 2008; Dib \& Kaspi 2014). Overall, the PSR J1119-6127 event is clearly magnetar-like.

Most similar to the PSR J1119-6127 event is the 2006 magnetar-like outburst of PSR J1846-0258 (Gavriil et al. 2008). The latter is also a young $(\tau<1000 \mathrm{yr})$, high-B $\left(B=5 \times 10^{13} \mathrm{G}\right)$ rotation-powered pulsar, albeit radio undetected (Archibald et al. 2008). Next we compare this object's 2006 outburst with the event studied here.

One difference between the outbursts is their energetics. For PSR J1119-6127, the $0.5-10-\mathrm{keV}$ flux as measured in the joint Swift and NuSTAR observation was $4.1 \times$ $10^{-11} \mathrm{erg} \mathrm{s}^{-1} \mathrm{~cm}^{-2}$. For a distance of $8.4 \mathrm{kpc}$, and assuming isotropic emission, this implies a luminosity of $3.5 \times$ $10^{35} \mathrm{erg} \mathrm{s}^{-1}$, or $0.1 \dot{E}$. This represents an increase over the quiescent value in this band of a factor of over $\sim 160$. Including the flux from $10 \mathrm{keV}$ extrapolated to the top of the NUSTAR band increases this value by $\sim 20 \%$. The efficiency for conversion of $\dot{E}$ to Fermi-band $\gamma$-ray emission, at least in quiescence, was estimated by Parent et al. (2011) to be 0.23 . With a comparable amount of energy suddenly appearing in X-rays, the Fermi-band emission may have been affected during this outburst. However, the normally low Fermi/LAT count rate (see \$3.1) requires multiple weeks of integration for a detection, hence a short-term anomaly may be undetectable.

By contrast, for PSR J1846-0258, in Chandra observations made within one week of its first detected magnetarlike burst, the unabsorbed flux in the $0.5-10-\mathrm{keV}$ band was $4.0 \times 10^{-11} \mathrm{erg} \mathrm{s}^{-1} \mathrm{~cm}^{-2}$ (Gavriil et al. 2008). For isotropic emission and a distance of $6 \mathrm{kpc}$ (Leahy \& Tian 2008), this implies a luminosity of $1.7 \times 10^{35} \mathrm{erg} \mathrm{s}^{-1}$, or $0.02 \dot{E}$. This represented an increase of a factor of 7.7 over the quiescent value. As this outburst had no realtime trigger, it is possible that initially the pulsar brightened more. However the pulsed flux measured by RXTE at the initial burst epoch was comparable a week later. Hence the increase in flux was probably not much larger than a factor of $\sim 10$, never exceeding a few percent of $\dot{E}$. Thus energetically, the PSR J1119-6127 outburst is far larger than that in PSR J1846-0258.

The spectral evolution in the two events also differed. In quiescence, PSR J1846-0258's X-ray spectrum is well described by a simple power law of index 1.2 (Gavriil et al. 2008), very different from the soft quiescent spectrum PSR J1119-6127. The latter, well described by a blackbody of $k T=0.2 \mathrm{keV}$ and power law of index 2.1 (Safi-Harb \& Kumar 2008; Ng et al. 2012), made this pulsar the youngest with detected thermal emission. It was also one of the hottest and most luminous thermal emitters even among rotationpowered pulsars of similar age (Olausen et al. 2013). This emission was also noted to be unusual for its high pulsed fraction (Ng et al. 2012).

During outburst, PSR J1846-0258 developed a bright soft component, whereas the harder power-law spectrum remained unchanged, apart from an increase in normalization by $\sim 35 \%$ above $10 \mathrm{keV}$ (Kuiper \& Hermsen 2009). The soft component was described as having a power-law spectrum of index 1.9 (Kumar \& Safi-Harb 2008; Gavriil et al. 2008). By contrast, the spectrum of PSR J1119-6127 has undergone a radical hardening, with a hot blackbody component and hard power law appearing. Nevertheless, the net effect in both outbursts was a transition to a spectrum very similar to those of bright magnetars.

It is also interesting to compare the outburst timing anomalies. In PSR J1846-0258, it suffered a sudden spin-up having $\Delta v / v \simeq 3 \times 10^{-6}$, followed by a large increase in $\dot{v}$ yielding a strong over-recovery of the glitch (Livingstone et al. 2010; Kuiper \& Hermsen 2009; Livingstone et al. 2011). The net long-term effect was a spin-down, accompanied by a change in braking index and a long-term enhancement in timing noise. While it is too early to know the post-outburst timing evolution in PSR J1119-6127, from our timing analysis, we find that the pulsar had a similar-sized spin-up glitch with $\Delta v / v \simeq 5.8 \times 10^{-6}$. Presently any increase in spin-down rate is modest compared to some glitch recoveries in magnetars and certainly compared to that following the 2006 PSR J1846-0258 glitch. However, greater evolution may yet be detected.

In young radio pulsars like PSR J1119-6127, hard X-ray emission is thought to arise in the context of outer gap models (e.g. Wang et al. 2013) from synchrotron radiation from secondary electron/positron pairs produced by inward propagating curvature radiation $\gamma$-rays. As discussed by Parent et al. (2011), in PSR J1119-6127, the X-ray/ $\gamma$-ray phase offset, together with the single-peak morphology of the $\gamma$-ray pulse, are well explained in outer gap models. The luminosity of both the X-ray and $\gamma$-ray emission in this picture must be bounded by the spin-down power.

The increase in X-ray luminosity particularly in the hard $\mathrm{X}$-ray range during the outburst of PSR J1846-0258 was argued by Kuiper \& Hermsen (2009) to be plausibly due to the above-described rotation-powered outer-gap emission, enhanced by particle injection due to perhaps to crust cracking that occurred at the glitch, reasonable given the lack of evidence for the hard X-ray luminosity exceeding more than a few percent of $\dot{E}$.

The new hard X-ray emission component in PSR J1119-6127 could have an outer-gap origin as well, but the large luminosity rise to within $0.1 \dot{E}$ in the X-ray band alone may be difficult to accommodate in such a picture, and might require a commensurate increase in $\gamma$-ray luminosity, impos- 
sible given the available $\dot{E}$ energy budget.

Alternatively, the hard X-ray emission may be magnetarlike. The origin of the bright hard X-ray component in magnetars has been argued to be a decelerating electron/positron flow in the closed magnetosphere, in the higher altitude regions of large magnetic loops (Beloborodov 2013). This emission is powered ultimately by the internal stellar magnetic field and is not limited by $\dot{E}$. In this interpretation, the pulsar suffered an instability such that a significant twist in its field lines occurred, with highly relativistic particles $(\gamma>>$ 10 ) injected near the star where $B>>B_{Q E D}=4.4 \times 10^{13} \mathrm{G}$. If this is origin of the hard X-rays in PSR J1119-6127, then the true internal field of this pulsar is far higher than is inferred from its dipole component. This would support the argument for additional non-dipolar field components in apparently low-magnetic-field magnetars (Rea et al. 2010; Scholz et al. 2014b). One way to test this explanation is through modelling of the phase-resolved hard X-ray spectrum. This can yield constraints on the geometry of the emission region (e.g. Hascoët et al. 2014). Such constraints could then be compared with similar ones from radio polarimetry (Weltevrede et al. 2011) and/or modelling of the $\gamma$-ray light curve (Parent et al. 2011).

Importantly, PSRs J1846-0258 and now J1119-6127 are the only two rotation-powered pulsars to have exhibited radiative changes at glitch epochs; this must be a consequence of their high spin-inferred $B$. Indeed no X-ray enhancement was seen in Chandra observations made 3.5 days following a large $\left(\Delta v / v=3 \times 10^{-6}\right)$ spin-up glitch in the lower-field $\left(B=3.4 \times 10^{12} \mathrm{G}\right)$ Vela radio pulsar (Helfand et al. 2001). On the other hand, there was no evidence for an X-ray enhancement in PSR J1846-0258 near the epoch of a much smaller glitch having $\Delta v / v=2.5 \times 10^{-9}$ (Livingstone et al. 2006), nor in previous glitches $\left(\Delta v / v=2.9 \times 10^{-7}\right.$ and $4.1 \times 10^{-6}$ in 2004 and 2007, respectively) in PSR J1119-6127 (Weltevrede et al. 2011; Antonopoulou et al. 2015), although prompt Xray observations were not performed in those cases. More- over, multiple sizeable glitches in bona fide magnetars have been unaccompanied by radiative changes ( $\mathrm{Scholz}$ et al. 2014a; Dib \& Kaspi 2014). This may indicate that an independent parameter such as the crustal depth of the glitch location plays a role in the radiative detectability of high-B neutron-stars at glitch epochs (see, e.g., Eichler \& Cheng 1989; Lyubarsky et al. 2002).

The possibility of a magnetar-like outburst from a high-B radio pulsar was discussed by Kaspi \& McLaughlin (2005), who also suggested the possibility of radio emission from magnetars prior to its discovery by Camilo et al. (2006). Perna \& Pons (2011) and Pons \& Perna (2011) provided theoretical groundwork for the hypothesis, and magnetothermal modelling such as that by Viganò et al. (2013) have further developed these ideas, which are now on solid observational ground. Other high-B radio pulsars like PSRs J1718-3718 (Zhu et al. 2011) and J1734-3333 (Olausen et al. 2013) seem likely to also undergo a magnetar-like transition in coming years.

Acknowledgements: The authors thank the operations teams of NUSTAR, particularly Karl Forster, and Swift for their speed and flexibility scheduling these observations. We thank Fermi-LAT Collaboration for the public data and tools used in this work. This work made use of data from the NUSTAR mission, a project led by the California Institute of Technology, managed by the Jet Propulsion Laboratory, and funded by the NASA. We acknowledge the use of public data from the Swift data archive. R.F.A. acknowledges support from an NSERC CGSD. V.M.K. receives support from an NSERC Discovery Grant, an Accelerator Supplement and from the Gerhard Herzberg Award, an R. Howard Webster Foundation Fellowship from the Canadian Institute for Advanced Study, the Canada Research Chairs Program, and the Lorne Trottier Chair in Astrophysics and Cosmology. S.P.T acknowledges support from a McGill Astrophysics postdoctoral fellowship.

\section{REFERENCES}

Antonopoulou, D., Vasilopoulos, G., \& Espinoza, C. M. 2016, ATel, 9282

Antonopoulou, D., Weltevrede, P., Espinoza, C. M., et al. 2015, MNRAS, 447, 3924

Archibald, A. M., Kaspi, V. M., Livingstone, M. A., \& McLaughlin, M. A. 2008, ApJ, 688, 550

Arnaud, K. A. 1996, in Astronomical Data Analaysis Software and Systems V, ed. G. Jacoby \& J. Barnes, Vol. 101 (San Fransisco: ASP), 17

Atwood, W. B., Abdo, A. A., Ackermann, M., et al. 2009, ApJ, 697, 1071

Beloborodov, A. M. 2013, ApJ, 762, 13

Burgay, M., Possenti, A., Kerr, M., et al. 2016, ATel, 9286

Burrows, D. N., Hill, J. E., Nousek, J. A., et al. 2005, Space Sci. Rev., 120, 165

Camilo, F., Kaspi, V. M., Lyne, A. G., et al. 2000, ApJ, 541, 367

Camilo, F., Ransom, S., Halpern, J., et al. 2006, Nature, 442, 892

Cash, W. 1979, ApJ, 228, 939

Caswell, J. L., McClure-Griffiths, N. M., \& Cheung, M. C. M. 2004, MNRAS, 352, 1405
Crawford, F., Gaensler, B. M., Kaspi, V. M., et al. 2001, ApJ, 554, 152

Dib, R., \& Kaspi, V. M. 2014, ApJ, 784, 37

Dib, R., Kaspi, V. M., \& Gavriil, F. P. 2008, ApJ, 673, 1044

Eichler, D., \& Cheng, A. F. 1989, ApJ, 336, 360

Gavriil, F. P., Gonzalez, M. E., Gotthelf, E. V., et al. 2008, Science, 319, 1802

Gonzalez, M., \& Safi-Harb, S. 2003, ApJ, 591, L143

Harrison, F. A., Craig, W. W., Christensen, F. E., et al. 2013, ApJ, 770, 103

Hascoët, R., Beloborodov, A. M., \& den Hartog, P. R. 2014, ApJL, 786, L1

Helfand, D. J., Gotthelf, E. V., \& Halpern, J. P. 2001, ApJ, 556, 380

Hobbs, G. M., Edwards, R. T., \& Manchester, R. N. 2006, MNRAS, 369, 655

Kargaltsev, O., Kouveliotou, C., Pavlov, G. G., et al. 2012, ApJ, 748, 26

Kaspi, V. M., \& McLaughlin, M. A. 2005, ApJ, 618

Kennea, J. A., Lien, A. Y., Marshall, F. E., et al. 2016, GCN, 19735

Kuiper, L., \& Hermsen, W. 2009, A\&A, 501, 1031

Kumar, H. S., \& Safi-Harb, S. 2008, ApJ, 678, L43 
Leahy, D. A., \& Tian, W. W. 2008, A\&A, 480, L25

Livingstone, M. A., Kaspi, V. M., \& Gavriil, F. P. 2010, ApJ, 710, 1710

Livingstone, M. A., Kaspi, V. M., Gotthelf, E. V., \& Kuiper, L. 2006, ApJ, 647, 1286

Livingstone, M. A., Ng, C.-Y., Kaspi, V. M., Gavriil, F. P., \& Gotthelf, E. V. 2011, ApJ, 730, 66

Livingstone, M. A., Ransom, S. M., Camilo, F., et al. 2009, ApJ, 706, 1163

Lyubarsky, Y., Eichler, D., \& Thompson, C. 2002, ApJ, 580, L69

Ng, C.-Y., Kaspi, V. M., Ho, W. C. G., et al. 2012, ApJ, 761, 65

Olausen, S. A., Zhu, W. W., Vogel, J. K., et al. 2013, ApJ, 764, 1

Parent, D., Kerr, M., den Hartog, P. R., et al. 2011, ApJ, 743

Perna, R., \& Pons, J. A. 2011, ApJL, 727, L51

Pons, J. A., \& Perna, R. 2011, ApJ, 741, 123

Ray, P. S., Kerr, M., Parent, D., et al. 2011, ApJS, 194, 17
Rea, N., \& Esposito, P. 2011, Astrophysics and Space Science Proceedings, 21, 247

Rea, N., Esposito, P., Turolla, R., et al. 2010, Science, 330, 944

Safi-Harb, S., \& Kumar, H. S. 2008, ApJ, 684, 532

Scholz, P., Archibald, R. F., Kaspi, V. M., et al. 2014a, ApJ, 783, 99

Scholz, P., \& Kaspi, V. M. 2011, ApJ, 739, 94

Scholz, P., Kaspi, V. M., \& Cumming, A. 2014b, ApJ, 786, 62

Viganò, D., Rea, N., Pons, J. A., et al. 2013, MNRAS, 434, 123

Wang, Y., Takata, J., \& Cheng, K. S. 2013, ApJ, 764, 51

Weltevrede, P., Johnston, S., \& Espinoza, C. M. 2011, MNRAS, 411, 1917

Younes, G., Kouveliotou, C., \& Roberts, O. 2016, GCN, 19736

Zhu, W. W., Kaspi, V. M., McLaughlin, M. A., et al. 2011, ApJ, 734, 44 